\title{
Chronic kidney disease of unknown origin in agricultural communities: beyond tropical mist?
}

\author{
Claire Rigothier $^{1,2}$, Bertrand Chauveau (D) ${ }^{3}$, Sébastien Rubin (D) ${ }^{1,4}$ and Christian Combe (D) ${ }^{1,2}$ \\ ${ }^{1}$ Service de Néphrologie, Transplantation, Dialyse et Aphérèses, CHU de Bordeaux, Bordeaux, France, ${ }^{2}$ Tissue Bioengineering Unit BioTis, \\ INSERM U1026, Bordeaux, France, ${ }^{3}$ Service de Pathologie, CHU de Bordeaux, Bordeaux, France and ${ }^{4}$ Biologie des Maladies Cardio-Vasculaires, \\ University of Bordeaux, INSERM U1034, Pessac, France
}

Correspondence to: Christian Combe; E-mail: christian.combe@chu-bordeaux.fr

In the last three decades, a peculiar type of tubulointerstitial nephritis has been described in agricultural populations, named Mesoamerican nephropathy (MeN) or Chronic Interstitial Nephritis in Agricultural Communities (CINAC) [1]. CINAC is a chronic tubulointerstitial nephropathy occurring generally in young men, mainly in sugarcane workers, in the absence of diabetes mellitus, hypertension, glomerular disease or other causes of renal disease, with no or minimal proteinuria $(<1 \mathrm{~g} /$ day) and minor urinary sediment abnormalities. Apart from the geographical origin of the patients and their occupational exposure, this definition is quite loose and could be applied to other forms of chronic interstitial nephritis such as analgesic nephropathy, aristolochic acid nephropathy, etc. Therefore, classifying chronic kidney disease (CKD) of unknown aetiology as CINAC might be challenging.

CINAC has been described in rural agricultural communities with low socioeconomic status, in different regions of the world: Central America, Sri Lanka and India [2, 3], and more recently, other patients with potential CINAC have been reported in countries such as Egypt, Morocco and even Portugal and France, that is, wealthier countries with a less hot climate [4]. There are currently controversies about the pathogenesis of CINAC, which could be linked to exposure to toxic products such as herbicides (glyphosate and its metabolites being one potential culprit), heavy metals, infections or high altitude, all of them in the context of intense heat [1-7], although this may not be always the case since patients have been identified in regions with a temperate climate [4].

In this issue of NDT, Aguilar-Ramirez et al. [8] have evaluated the prevalence of CKD in a middle-aged population (42 years, interquartile range 31-50) in Central America (Tierra Blanca, Mexico). They have chosen this rural region because of its hot tropical climate, with a mean annual temperature of $31^{\circ} \mathrm{C}$, and extremes as high as $45^{\circ} \mathrm{C}$, and because they had preliminary warnings about a high $\mathrm{CKD}$ incidence there. As a matter of fact, they have found that $3.3 \%(n=19)$ of the population had estimated glomerular filtration rate (eGFR) $<60 \mathrm{~mL} / \mathrm{min} /$ $1.73 \mathrm{~m}^{2}$, and there was a very high $(24.7 \%)$ prevalence of probable $\mathrm{CKD}$, mainly accounted by elevated albumin/creatinine ratio. Approximately one-quarter of the studied population had an occupation in agriculture, with an exposure to agrochemicals in $27.6 \%$. Statistical analyses showed that 'working in agriculture' was strongly associated with the risk of having an eGFR $<60 \mathrm{~mL} / \mathrm{min} / 1.73 \mathrm{~m}^{2}$ [8], when adjustments were made for classical CKD-associated factors including age, sex, obesity, diabetes, hypertension, education and residence (odds ratio 5.19, 95\% confidence interval 1.11-24.26).

This is an important study in the context of $\mathrm{MeN}$, for which the authors should be congratulated, some important strengths being the choice of rural regions, the exhaustivity of the population studied and the use of a central lab. On the other hand, this study illustrates the difficult challenges to identifing potential causes of CKD in this population. (i) A first challenge is the number of patients studied. In this study, although 579 persons were included, only 19 had an eGFR $<60 \mathrm{~mL} / \mathrm{min} / 1.73 \mathrm{~m}^{2}$ [8], which is a low number for performing multivariate analyses. (ii) A second point is that only one determination of biological indicators of CKD was performed, which could be considered as insufficient to confirm the chronic nature of CKD. (iii) Economic status is missing from the sociodemographic parameters, although many studies have shown in several populations an association between poverty and CKD; this is a crucial point in a population with potential difficulties in access to care. (iv) The authors claim that there was no association between education level and CKD, but when 'Elementary education' and 'No formal education' are merged, there is a significant association between low-level education and eGFR $\leq 60 \mathrm{~mL} / \mathrm{min} / 1.73 \mathrm{~m}^{2}$ ( $\mathrm{P}<0.05$ by Fisher's exact test). (v) 'Occupation' was asked as the main one during the last 12 months [8]. One could argue that the deleterious effects on the kidneys of working in agriculture and being exposed to agrochemicals might require more time, and that a more detailed and longer evaluation of occupation and exposure to potential toxics should have been performed [7]. (vi) It is remarkable that $18.0 \%$ of the participants reported a family history of kidney disease, and it could be speculated that some genetic factors, including hypertension and Type 2 diabetes, could be involved in the high prevalence of CKD in this population [9]. (vii) Nearly $40 \%$ of the population 
were reported to have had recurrent urinary tract infections (67\% of the participants were women), another potential factor of chronic interstitial nephritis.

When one considers all the above questions, it is quite difficult to have a clear idea of the factors associated with CKD in this population, and of the potential role of agriculture and environmental factors. Pathophysiological mechanisms leading to CINAC need to be elucidated: De Broe's group has recently depicted abnormal lysosomes in proximal tubules of patients suffering from CINAC [4], including patents from our institution. These modifications were also observed in kidney transplant recipients treated by calcineurin inhibitors, in rats exposed to calcineurin inhibitors but also in patients with other drug exposures such as lithium. In De Broe's hypothesis, CINAC seems to be a toxin-induced lysosomal tubulopathy involving the calcineurin pathway.

Despite uncertainties of the mechanism involved in the pathophysiology of CINAC, its prevention is based on the eviction of toxic pesticides, abundant water hydration (with a specific attention to weight) and medical surveillance (dipstick and measurement of serum creatinine and electrolytes). A detailed survey of occupational and environmental parameters is required to confirm CINAC diagnosis and the risk factors associated. Some therapeutics (metformin) might be useful to limit the agrochemical toxicities, but randomized controlled studies are needed.

\section{CONFLICT OF INTEREST STATEMENT}

None declared.

\section{REFERENCES}

1. Johnson RJ, Wesseling C, Newman LS. Chronic kidney disease of unknown cause in agricultural communities. N Engl J Med 2019; 380: 1843-1852

2. Abraham G, Agarwal SK, Gowrishankar S et al. Chronic kidney disease of unknown etiology: hotspots in India and other Asian countries. Semin Nephrol 2019; 39: 272-277

3. Wijkström J, Leiva R, Elinder C-G et al. Clinical and pathological characterization of Mesoamerican nephropathy: a new kidney disease in Central America. Am J Kidney Dis 2013; 62: 908-918

4. Vervaet BA, Nast CC, Jayasumana C et al. Chronic interstitial nephritis in agricultural communities is a toxin-induced proximal tubular nephropathy. Kidney Int 2020; 97: 350-369

5. Correa-Rotter R, García-Trabanino R. Mesoamerican nephropathy. Semin Nephrol 2019; 39: 263-271

6. Herath C, Jayasumana C, De Silva PMCS et al. Kidney diseases in agricultural communities: a case against heat-stress nephropathy. Kidney Int Rep 2018; 3: 271-280

7. Lebov JF, Engel LS, Richardson D et al. Pesticide use and risk of end-stage renal disease among licensed pesticide applicators in the Agricultural Health Study. Occup Environ Med 2016; 73: 3-12

8. Aguilar-Ramirez D, Raña-Custodio A, Villa A et al. Decreased kidney function and agricultural work: a cross-sectional study in middle-aged adults from Tierra Blanca, Mexico. Nephrol Dial Transplant 2020; 142: 746. doi: 10.1093/ndt/gfaa041

9. Wuttke M, Kottgen A. Insights into kidney diseases from genome-wide association studies. Nat Rev Nephrol 2016; 12: 549-562

Received: 25.8.2020; Editorial decision: 27.9.2020 